\title{
Spin- and valley-dependent miniband structure and transport in silicene superlattices
}

\author{
N. Missault, ${ }^{1, *}$ P. Vasilopoulos,${ }^{2, \dagger}$ F. M. Peeters,${ }^{1, \ddagger}$ and B. Van Duppen ${ }^{1, \S}$ \\ ${ }^{1}$ Departement Fysica, Universiteit Antwerpen Groenenborgerlaan 171, B-2020 Antwerpen, Belgium \\ ${ }^{2}$ Department of Physics, Concordia University, 7141 Sherbrooke West, Montreal, Quebec, Canada H4B 1 R6
}

(Received 13 January 2016; published 21 March 2016)

\begin{abstract}
We investigate silicene superlattices in the presence of a tunable barrier potential $U$, an exchange field $M$, and a perpendicular electric field $E_{z}$. The resulting miniband structure depends on the spin and valley indices and on the fields $M$ and $E_{z}$. These fields determine the minigaps and also affect the additional Dirac points brought about by the periodic potential $U$. In addition, we consider diffusive transport and assess its dependence on the spin and valley indices as well as on temperature. The corresponding spin and valley polarizations strongly depend on the potential $U$ and can be made almost $100 \%$ at very low temperatures at particular values of the Fermi energy.
\end{abstract}

DOI: 10.1103/PhysRevB.93.125425

\section{INTRODUCTION}

Silicene is a two-dimensional (2D) hexagonal lattice of silicon atoms, similar in structure to graphene, except for the fact that its two sublattices have a height difference of $2 \ell$ with the buckling height $\ell \approx 0.23 \AA$. This material has attracted considerable attention in recent years [1]. It has been predicted to be stable [2] and there have been several attempts to synthesize it [3-6]. The energy spectrum of silicene has a small gap due to its strong spin-orbit interaction [7], determined by the coupling strength $\lambda_{s o}$. Due to the buckled structure of silicene, this gap can be tuned by applying a perpendicular electric field [8,9], leading to a gap of size $2\left(s_{z} \eta E_{z} \ell \pm \lambda_{s o}\right)$, where $s_{z}= \pm 1$ and $\eta= \pm 1$ are the spin and valley indices, respectively. This tuning of the gap, a property which is absent in graphene, and its compatibility with silicon-based technology, makes silicene an excellent candidate for applications in nanoelectronics. Very recently, a silicene based field-effect transistor was reported [10].

One can also induce an exchange field $M$ in silicene by putting a ferromagnet near its surface and further influence its spin properties. Combined with the spin and valley dependence of the gap and the fact that silicon has a longer spindiffusion time [11,12] and spin-coherence length [13] than graphene [14], this makes silicene an excellent candidate for applications in spintronics and valleytronics. Such considerations led to many studies of important effects such as the spinand valley-Hall effects [15-18], the quantum anomalous Hall effect $[9,19]$, the spin-valley coupling [20], etc., see Ref. [21] for a review.

Recent studies on the influence of electric and exchange fields on ballistic transport through potential barriers led to the prediction of near-perfect spin $p_{s}$ and valley $p_{v}$ polarizations [22-24] for energies near the barrier height $U$. Also, $p_{s}$ and $p_{v}$ increase with the number of barriers [24]. Such findings strongly motivate studying silicene superlattices (SSLs) and the influence of scattering on the corresponding, nonballistic transport. It is also of interest to investigate how

\footnotetext{
*nathan.missault@uantwerpen.be

†p.vasilopoulos@concordia.ca

†francois.peeters@uantwerpen.be

§ben.vanduppen@uantwerpen.be
}

the additional Dirac points, caused by the SL potential as shown for graphene $[25,26]$ with $E_{z}=M=0$, are affected by finite $E_{z}$ and $M$. The condition for these extra Dirac points is $\sin \left(q_{x} b\right)=0$, where $q_{x}$ is the $x$ component (we take $x$ perpendicular to the barriers as depicted in Fig. 1) of the wave vector inside the barriers of width $b$; it means that half of the particle's wavelength fits an integer number of times inside the barrier.

The aim of the present research is to build on recent studies [23,24] and study SSLs. In particular, the aim is to assess the dependence of the miniband structure on the spin and valley degrees of freedom and on the fields $M$ and $E_{z}$. A second aim is to go beyond the ballistic transport of Refs. [23,24] and investigate the more realistic diffusive transport, its dependence on these factors, and evaluate $p_{s}$ and $p_{v}$ at finite temperature. The main findings of this work are as follows: (i) the positions of the additional Dirac points found near the $K\left(K^{\prime}\right)$ valley in the energy spectrum depend on the fields $E_{z}$ ad $M$ since so does the $x$ component of the wave vector in the barriers and wells; (ii) the minigaps initially increase with $E_{z}$ and $M$ but with further increase they close. This behavior of the energy spectrum is reflected in the density of states and the conductivity, which are spin and valley dependent, and the spin $p_{s}$ and valley $p_{v}$ polarizations. (iii) At very low temperatures $p_{s}$ and $p_{v}$ are nearly perfect, within a realistic range of the fields $E_{z}$ and $M$, but decrease as the temperature is raised. iv) Contrary to results for a finite number of barriers [22-24], in the ballistic regime these polarizations are no longer restricted to energies near the top of the barriers but do depend on $U$.

The paper is organized as follows. In Sec. II we present the basics of the formalism and obtain the SL dispersion relation. In Sec. III we present results for the miniband structure, the density of states, and the diffusive conductivity. In addition, we evaluate $p_{s}$ and $p_{v}$ and assess their dependence on the fields $M$ and $E_{z}$ and the temperature. We summarize our main results in Sec. IV.

\section{DISPERSION RELATION}

We consider silicene in the presence of a SL potential $U$, an exchange field $M$, and a perpendicular electric field $E_{z}$. The field $M$ can be induced by placing a ferromagnetic layer near the surface of silicene. We assume that the different spin 


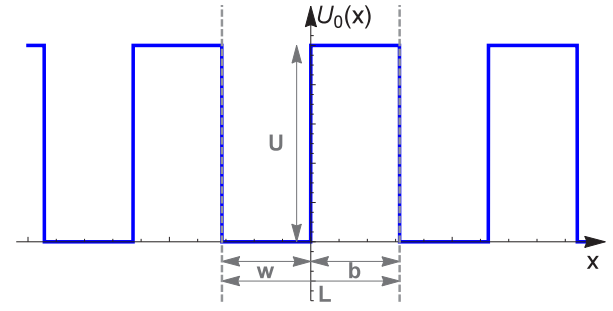

FIG. 1. Sketch of the superlattice potential.

states are completely decoupled, which means we neglect very small intrinsic and extrinsic Rashba effects referred to as $\lambda_{R 1}$ and $\lambda_{R 2}$ in Refs. [9,27]. Then for low energies the one-electron Hamiltonian is given by

$$
H_{\eta, s_{z}}=\left(\begin{array}{cc}
\Delta_{z}+U(x) & \hbar v_{F}\left(k_{x}+i \eta k_{y}\right) \\
\hbar v_{F}\left(k_{x}-i \eta k_{y}\right) & -\Delta_{z}+U(x)
\end{array}\right) .
$$

Here $v_{F}$ is the Fermi velocity, $U(x)=U_{0}(x)+s_{z} M$ is the potential energy function, which includes the periodic superlattice potential $U_{0}(x)$ and the exchange field $M$, and $\Delta_{z}=s_{z} \eta e E_{z} l+\lambda_{s o}$ contains the electric field strength $E_{z}$, the buckling height $l$, and the spin-orbit interaction (SOI) strength $\lambda_{s o}$. The indices $\eta$ and $s_{z}$ distinguish, respectively, between the $K$ and $K^{\prime}$ valleys and the spin-up $(\uparrow)$ and spin-down states $(\downarrow)$.

For $U(x)=0$ the energy spectrum is given by

$$
E_{s_{z}, \eta}= \pm\left(\left[\Delta_{z}^{2}+\hbar^{2} v_{F}^{2} k^{2}\right]^{1 / 2}+s_{z} M\right),
$$

which has a spin and valley dependent energy gap of size $2\left(\Delta_{z}+s_{z} M\right)$ and the spectrum is linear for larger $k$. With translational invariance in the $y$ direction the eigenstates outside the barriers are $\Psi(x, y)=\psi(x) e^{i k_{y} y}$ where

$$
\psi(x)=A\left(\begin{array}{c}
1 \\
r k_{-}
\end{array}\right) e^{i k_{x} x}+B\left(\begin{array}{c}
1 \\
-r k_{+}
\end{array}\right) e^{-i k_{x} x},
$$

with $r=\hbar v_{F} /\left(E-s_{z} M+\Delta_{z}\right), k_{ \pm}=k_{x} \pm i \eta k_{y}$, and

$$
k_{x}=\left[\left(\left(E-s_{z} M\right) / \hbar v_{F}\right)^{2}-\left(\Delta_{z} / \hbar v_{F}\right)^{2}-k_{y}^{2}\right]^{1 / 2}
$$

the $x$ component of the wave vector outside the barriers. Inside the barriers the wave functions are found by changing $E$ to $E-U_{0}$; for instance, the $x$ component of the wave vector is

$$
q_{x}=\left[\left(\left(E-U_{0}-s_{z} M\right) / \hbar v_{F}\right)^{2}-\left(\Delta_{z} / \hbar v_{F}\right)^{2}-k_{y}^{2}\right]^{1 / 2} .
$$

The SL structure has a unit cell of width $L$ which contains a barrier of width $b$, and a quantum well of width $w=L-b$, as depicted in Fig. 1. The wave functions obey Bloch's theorem and are continuous at the potential steps. This leads to

$$
\begin{aligned}
\psi\left(0_{-}\right) & =\psi\left(0_{+}\right) \\
\psi(b) & =\psi(-w) e^{i \kappa_{x} L} .
\end{aligned}
$$

After matching the wave functions and applying the conditions (6) we obtain the SL dispersion relation

$$
\cos \left(\kappa_{x} L\right)=\cos \left(w k_{x}\right) \cos \left(b q_{x}\right)-F \sin \left(w k_{x}\right) \sin \left(b q_{x}\right)
$$

with

$$
\begin{aligned}
F & =\frac{k_{x}^{2} \epsilon_{b}^{2}+q_{x}^{2} \epsilon_{w}^{2}+k_{y}^{2}\left(\epsilon_{b}-\epsilon_{w}\right)^{2}}{2 k_{x} q_{x} \epsilon_{w} \epsilon_{b}} \\
\epsilon_{w} & =E+\Delta_{z}, \quad \epsilon_{b}=E+\Delta_{z}-U_{0}-s_{z} M .
\end{aligned}
$$

Apart from the explicit dependence on the spin and valley indices as well as the fields $M$ and $E_{z}$, Eq. (7) is of the standard form and has to be solved numerically.

\section{RESULTS AND DISCUSSION}

\section{A. Spectrum, density of states, and conductivity}

It is convenient to introduce new units which results in the following dimensionless quantities: $E \rightarrow \epsilon=\left(E / \hbar v_{F}\right) L$, $U \rightarrow u=\left(U / \hbar v_{F}\right) L, \Delta_{z} \rightarrow\left(\Delta_{z} / \hbar v_{F}\right) L, \kappa_{x} \rightarrow \kappa_{x} L, k_{y} \rightarrow$ $k_{y} L, w \rightarrow w / L, b \rightarrow b / L$. Additionally, we will substitute the energy between the barriers by $\epsilon \rightarrow \epsilon+b u$ and within the barriers by $\epsilon-u \rightarrow \epsilon-w u$, in order to avoid shifting the Fermi energy by $u / 2$ as a result of adding the superlattice potential to the system. By solving Eq. (7) numerically we find the energy spectrum shown in Figs. 2 and 3. It is periodic in $\kappa_{x}$ and exhibits a miniband structure. For low values of $u$, it is mostly hyperbolic as a function of $k_{y}$. However, as we see in Fig. 3, the structure changes significantly for higher values of $u$. For $u>4 \pi$, there are two extra Dirac points for $\kappa_{x}=0$, and even more for higher values of $u$. The condition for them is easily found by solving Eq. (7) for $\epsilon=0, w=b=0.5$, and $\kappa_{x}=0$. For $w \neq b$ these extra Dirac points do not occur at

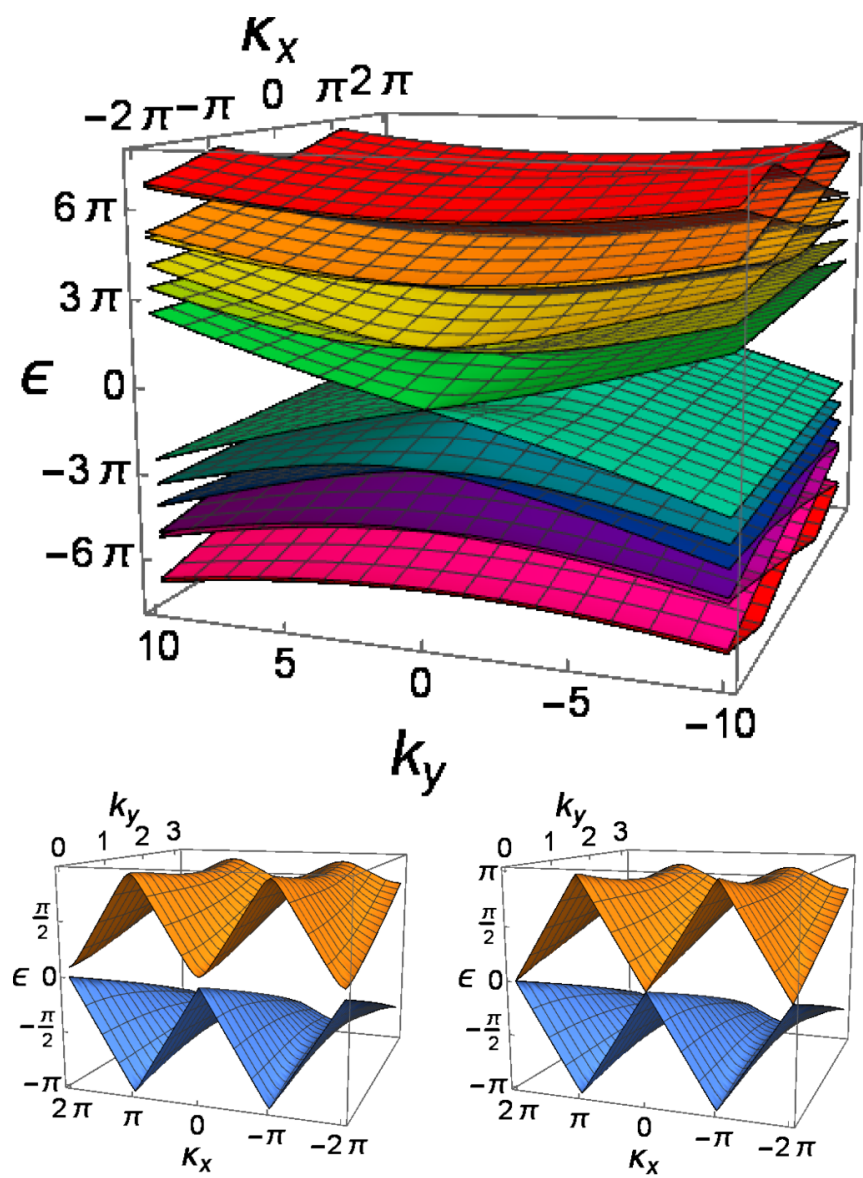

FIG. 2. Upper panel: Superlattice miniband structure with $\Delta_{z}=$ $2 \lambda_{s o}$. Lower panels: Miniband structure for spin-down electrons (left) and for spin-up electrons (right) near the K valley. Near the $\mathrm{K}^{\prime}$ valley the results for spins down and spins up are reversed. The parameters used are $u=2 \pi, b=w=0.5, e E z \ell=\lambda_{s o}$ and $M=0$. 

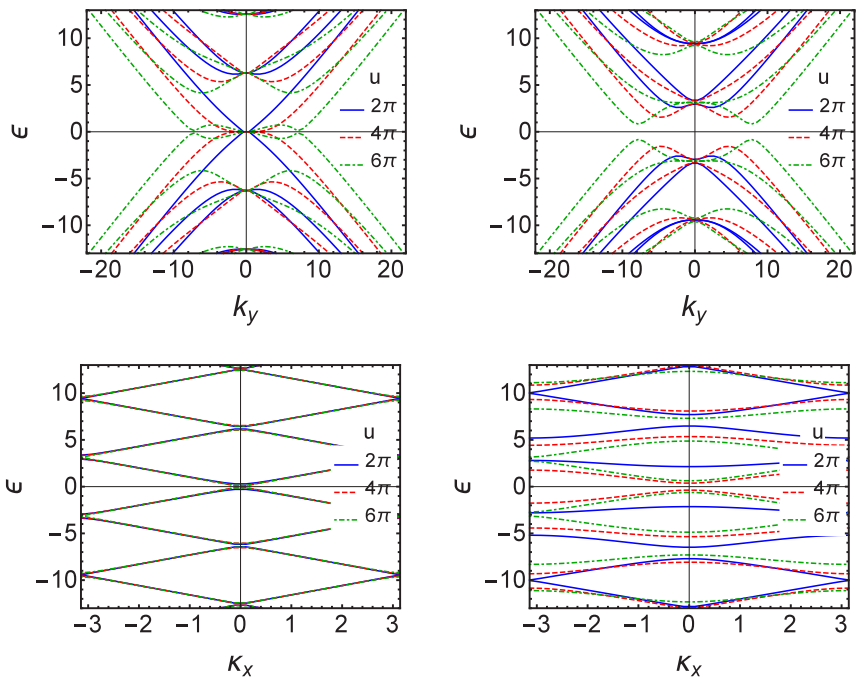

FIG. 3. Miniband structure for $b=w=0.5$ and $\Delta_{z}=2 \lambda_{s o}$. The top left (right) panel is for $\kappa_{x}=0\left(\kappa_{x}=\pi\right)$, and the bottom left (right) one for $k_{y}=0\left(k_{y}=\pi\right)$.

$\epsilon=0$ but at $\epsilon \neq 0$, see Ref. [26] for more details. For $\epsilon=0$ we find

$$
0=\left(\frac{u^{2}+4\left(\Delta_{z}^{2}+k_{y}^{2}\right)}{u^{2}-4\left(\Delta_{z}^{2}+k_{y}^{2}\right)}-1\right) \sin ^{2}\left(k_{x} / 2\right) .
$$

For $k_{y} \neq 0$ or $\Delta_{z} \neq 0$, Eq. (10) leads to $\sin \left(k_{x} / 2\right)=0$. Its solution corresponds to the original Dirac point when a critical field value $E_{c}= \pm \lambda_{\text {so }} / e \ell$ closes the gap. In turn $\sin \left(k_{x} / 2\right)=0$ leads to

$$
k_{y}=\left[(u / 2)^{2}-(2 j \pi)^{2}-\Delta_{z}^{2}\right]^{1 / 2}
$$

where $j$ is an integer larger than 0 . For $\Delta_{z}=0$ Eq. (11) reduces to the result for graphene [26]. The condition $\sin \left(k_{x} / 2\right)=0$ expresses the fact that half the wavelength $\lambda=2 \pi / k_{x}\left(2 \pi / q_{x}\right)$ fits an integer number of times inside the barriers (wells). Note however that this is not the case for the Bloch wave vector $\kappa_{x}$. The behavior of the energy spectrum at $\kappa_{x}=k_{y}=0$ as a function of $k_{x}$ is shown in Fig. 4. The upper panels are for different $E_{z}$ with $M$ fixed and the lower ones for different $M$ with $E_{z}$ fixed. The exchange field $M$ simply raises (lowers) the energy of spin-down (spin-up) electrons. As seen the minigaps increase with $E_{z}$. After reaching a maximum size though, they do decrease as shown for the first two of them in Fig. 5. The decrease occurs when two of the extra Dirac points are shifted to the $\mathrm{K}$ valley. The minigaps behave in a similar way when the exchange field $M$ is varied.

Using the SL miniband structure, we can find the density of states (DOS) given by

$$
D(\epsilon)=\sum_{n, \vec{k}} \delta\left(\epsilon-\epsilon_{n}(\vec{k})\right)
$$

where the integer $n$ labels the minibands and $\vec{k}=\kappa_{x} \vec{e}_{x}+k_{y} \overrightarrow{e_{y}}$. The results are shown in Fig. 6 for several values of $u$. Similar to the case of graphene [26], the DOS peaks at the edges of the Brillouin zones. These values correspond to local minima, maxima, or saddle points in the energy spectrum, which have different positions in the $k$ plane for different values of $u$.
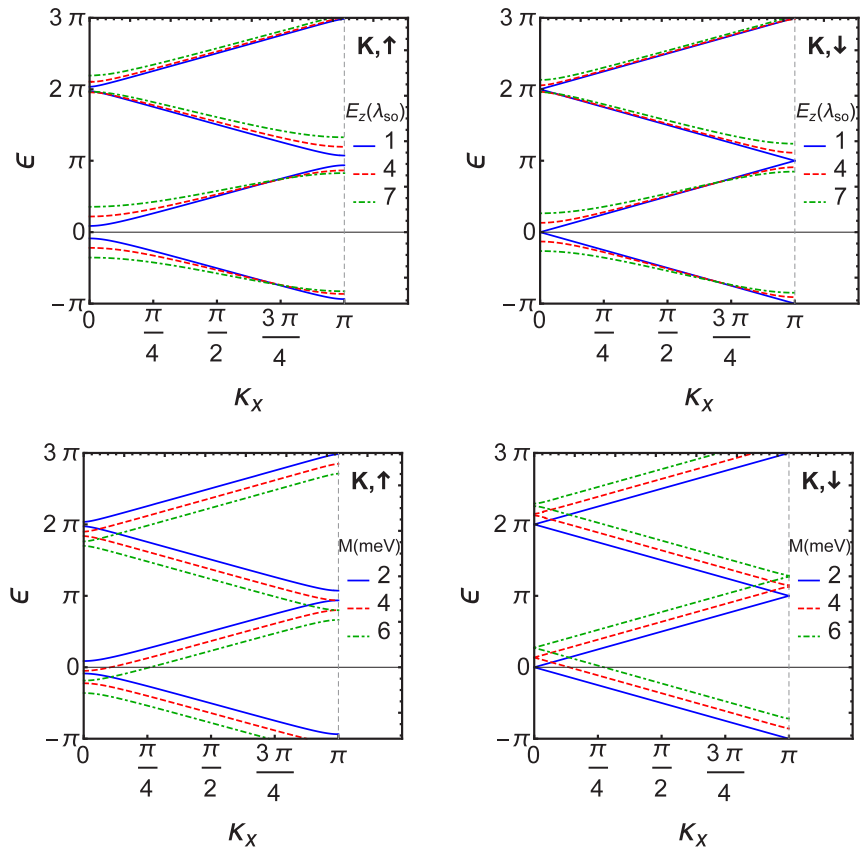

FIG. 4. Miniband structure for spin-up (left) and spin-down (right) electrons near the $K$ valley at $k_{y}=0$ for three values of the field $E_{z}$ (top row) and of the field $M$ (bottom row).

Near these points the energy states accumulate. It is also clear that for higher potential barriers the peaks get larger as this corresponds to more pronounced minima of the energy as a function of $k_{y}$ as seen in Fig. 3.

The energy spectrum also allows us to calculate the diffusive conductivity $\sigma_{\mu \nu}$, with $\mu=x, y$ and $\nu=x, y$, which measures the system's capacity to carry a current in the $\mu$ direction in response to an electric field in the $v$ direction. If we assume a nearly constant relaxation time $\tau\left(E_{F}\right) \approx \tau_{F}$ the expression for $\sigma_{\mu \nu}$ is [28]

$$
\sigma_{\mu \nu}\left(\epsilon_{F}\right)=\beta \sigma_{0} \sum_{n, \mathbf{k}} v_{n \mu} v_{n v} f_{n \mathbf{k}}\left(1-f_{n \mathbf{k}}\right)
$$

with

$$
v_{n \mu}(\mathbf{k})=v_{F}\left[\partial \epsilon_{n}(\mathbf{k}) / \partial k_{\mu}\right]
$$

the group velocity in the $\mu$ direction, $\sigma_{0}=\tau_{F} e^{2} / A, A$ is the area of the system, $\beta=1 / k_{B} T, f_{n}(\mathbf{k})=1 /\left(e^{\beta\left(E_{F}-E_{n \mathbf{k}}\right)}+1\right)$ the Fermi-Dirac distribution, and $\tau$ a relaxation time for elastic
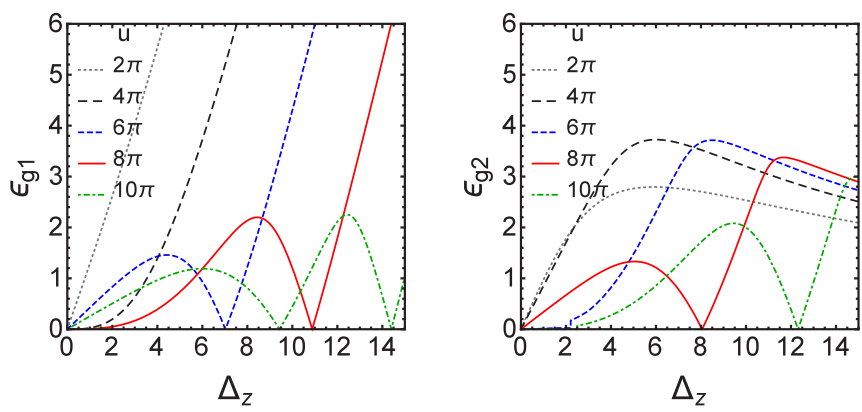

FIG. 5. Behaviour of the first (left) and second (right) gaps at the $K$ point as a function of $\Delta_{z}$. 


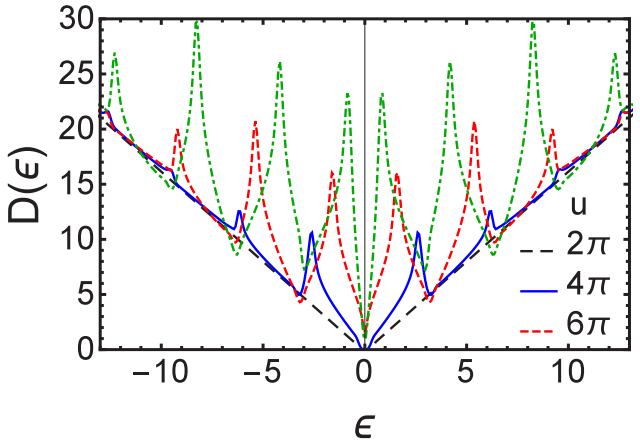

FIG. 6. Density of states for $w=b=0.5$ and $\Delta_{z}=2 \lambda_{s o}$. The $\delta$ function was approximated by a Gaussian with variance $\sigma^{2}=0.01$.

scattering. In the absence of a magnetic field we only consider $\mu=v$. However, there is a spin- and a valley-Hall effect, as discussed in Ref. [29]. The analytical expression for the group velocity in the $x$ direction is

$$
\begin{aligned}
\frac{v_{x}}{v_{F}}= & {\left[\sin \left(k_{x} w\right) \cos \left(q_{x} b\right)\left(\frac{\epsilon+u b}{k_{x}} w+F \frac{\epsilon-u w}{q_{x}} b\right)\right.} \\
& +\cos \left(k_{x} w\right) \sin \left(q_{x} b\right)\left(\frac{\epsilon-u w}{q_{x}} b+F \frac{\epsilon+u b}{k_{x}} w\right) \\
& \left.+F^{\prime} \sin \left(k_{x} w\right) \sin \left(q_{x} b\right)\right]^{-1} \sin \left(\kappa_{x} w\right),
\end{aligned}
$$

where

$$
\begin{aligned}
F^{\prime}= & \frac{(\epsilon+u b) \epsilon_{b}^{2}+(\epsilon-u w) \epsilon_{w}^{2}+k_{x}^{2} \epsilon_{b}+q_{x}^{2} \epsilon_{w}}{k_{x} q_{x} \epsilon_{w} \epsilon_{b}} \\
& +\frac{F}{2}\left(\frac{\epsilon+u b}{k_{x}^{2}}+\frac{\epsilon-u w}{q_{x}^{2}}+\frac{1}{\epsilon_{w}}+\frac{1}{\epsilon_{b}}\right) .
\end{aligned}
$$

The expression for $v_{y}$ is found in the same straightforward manner as Eq. (15) but the result is quite large and therefore will be omitted. The peaks in the conductivity $\sigma_{x x}$ (see Fig. 7) correspond to the peaks of the DOS at the Fermi energy and broaden as the temperature increases. For $T \rightarrow 0$ we can replace $\beta f_{n \mathbf{k}}\left(1-f_{n \mathbf{k}}\right)$ by $\delta\left(\epsilon_{F}-\epsilon_{n \mathbf{k}}\right)$ and obtain

$$
\sigma_{\mu \mu}\left(\epsilon_{F}\right)=\sigma_{0} v_{F} \sum_{n, i} \frac{v_{\mu}^{2}\left(\mathbf{k}_{\mathbf{i}}\right)}{\left[v_{x}^{2}\left(\mathbf{k}_{\mathbf{i}}\right)+v_{y}^{2}\left(\mathbf{k}_{\mathbf{i}}\right)\right]^{1 / 2}}
$$
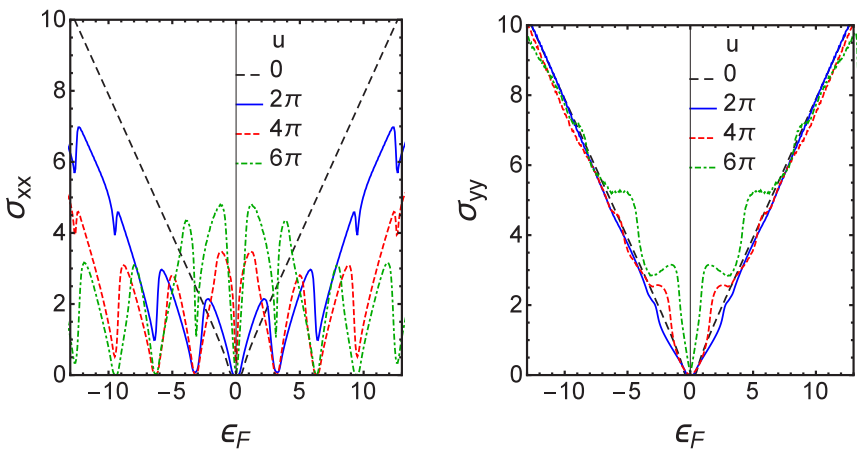

FIG. 7. Diffusive conductivity, in units of $\sigma_{0}$, along the $x$ (left) and $y$ (right) directions for $w=b=0.5, T=10 \mathrm{~K}$, and $E_{z}=2 \lambda_{\text {so }} / \mathrm{e} \ell$.
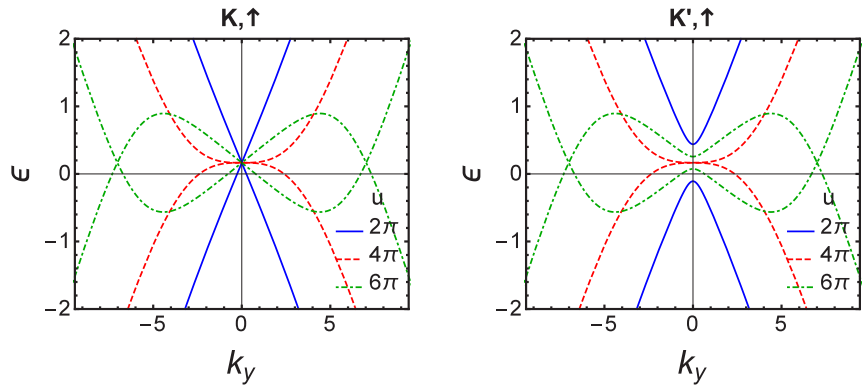

FIG. 8. Energy spectra for electrons with different valley degrees of freedom, with $M=1$ and $E_{z}=\lambda_{s o} / e \ell$.

where $i$ labels the solutions of $\epsilon_{n}\left(\mathbf{k}_{\mathbf{i}}\right)=\epsilon_{F}$. The conductivities $\sigma_{x x}$ and $\sigma_{y y}$ are shown in Fig. 7 for several values of $u$. As seen, for low energies $\sigma_{x x}$ is larger than without the SL potential $(u=0)$, while for higher energies the opposite is true. As for $\sigma_{y y}$, it is mostly linear except for large $u$, similar to the case for free silicene $(u=0)$, because of the translational invariance in the $y$ direction.

\section{B. Spin and valley polarizations}

Electrons with different spin and valley indices have a different energy gap for the same electric field $E_{z}$ and when the system is magnetized, the different spin states differ in energy, as shown in Fig. 8. It is clear that these differences are also present in the corresponding conductivities. Due to the lifted spin and valley degeneracies, we can define a spin polarization $p_{s}^{\mu}$ by

$$
p_{s}^{\mu}=\frac{\sigma_{\mu \mu}^{K \uparrow}+\sigma_{\mu \mu}^{K^{\prime} \uparrow}-\sigma_{\mu \mu}^{K \downarrow}-\sigma_{\mu \mu}^{K^{\prime} \downarrow}}{\sigma_{\mu \mu}^{K \uparrow}+\sigma_{\mu \mu}^{K^{\prime} \uparrow}+\sigma_{\mu \mu}^{K \downarrow}+\sigma_{\mu \mu}^{K^{\prime} \downarrow}}
$$

and a valley polarization $p_{v}^{\mu}$ by

$$
p_{v}^{\mu}=\frac{\sigma_{\mu \mu}^{K \uparrow}=\sigma_{\mu \mu}^{K^{\prime} \uparrow}+\sigma_{\mu \mu}^{K \downarrow}-\sigma_{\mu \mu}^{K^{\prime} \downarrow}}{\sigma_{\mu \mu}^{K \uparrow}+\sigma_{\mu \mu}^{K^{\prime} \uparrow}+\sigma_{\mu \mu}^{K \downarrow}+\sigma_{\mu \mu}^{K^{\prime} \downarrow}} .
$$

The results for $p_{s}^{\mu}$ and $p_{v}^{\mu}$ are shown in Fig. 9. One sees that they are optimal near $\epsilon_{F}=n \pi$ with $n$ an integer, i.e., at the location of the energy gaps. Since above and below the gaps they are opposite to each other, this allows for their quick switching by varying the electron energy. Near which gaps the polarizations are optimal depends on the potential strength $u$. As $u$ increases, the polarizations increase near the gaps
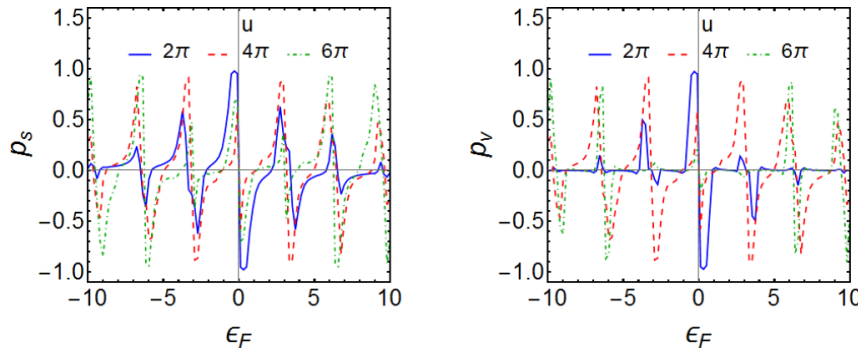

FIG. 9. Spin and valley polarization based on the conductivity in the $x$ direction for $w=b=0.5, e E_{z} l=5 \lambda_{s o}$, and $M=2 \mathrm{meV}$ for varying $u$. 
at higher energies, while near the primary gap they decrease. This corresponds to the increased conductivity visible in Fig. 7 , which is due to the extra Dirac points and the smaller energy gaps at high values of $u$. As the conductivity remains high for all electrons, the polarization is less drastic, which is why they are only optimal near the gaps in the first place. In the $y$ direction we only find a polarization near the zero-energy gap cases. Since this is not different from the results for silicene without the SL, we omit further details.

In Fig. 10 we show how the conductivity and the polarizations behave as a function of the fields $E_{z}$ and $M$. The conductivity increases as a function of $M$, since the electron energy moves away from the gap, and decreases as a function of $E_{z}$, because the field $E_{z}$ increases the gap to the point that the electron energy lies in the gap. The spin polarization is obviously an odd function of the magnetization but disappears for higher values of $M$, because the overall higher conductivity lowers the relative difference between the conductivity for different spins. A high valley polarization is only possible for a sufficiently strong electric field $E_{z}$. We also see that near the second energy gap the polarizations disappear for too strong fields $E_{z}$. This can be explained by the fact that the miniband gaps do not keep increasing for stronger fields, due to the further opening of the first gap, which squeezes the minibands closer together, see Fig. 5.
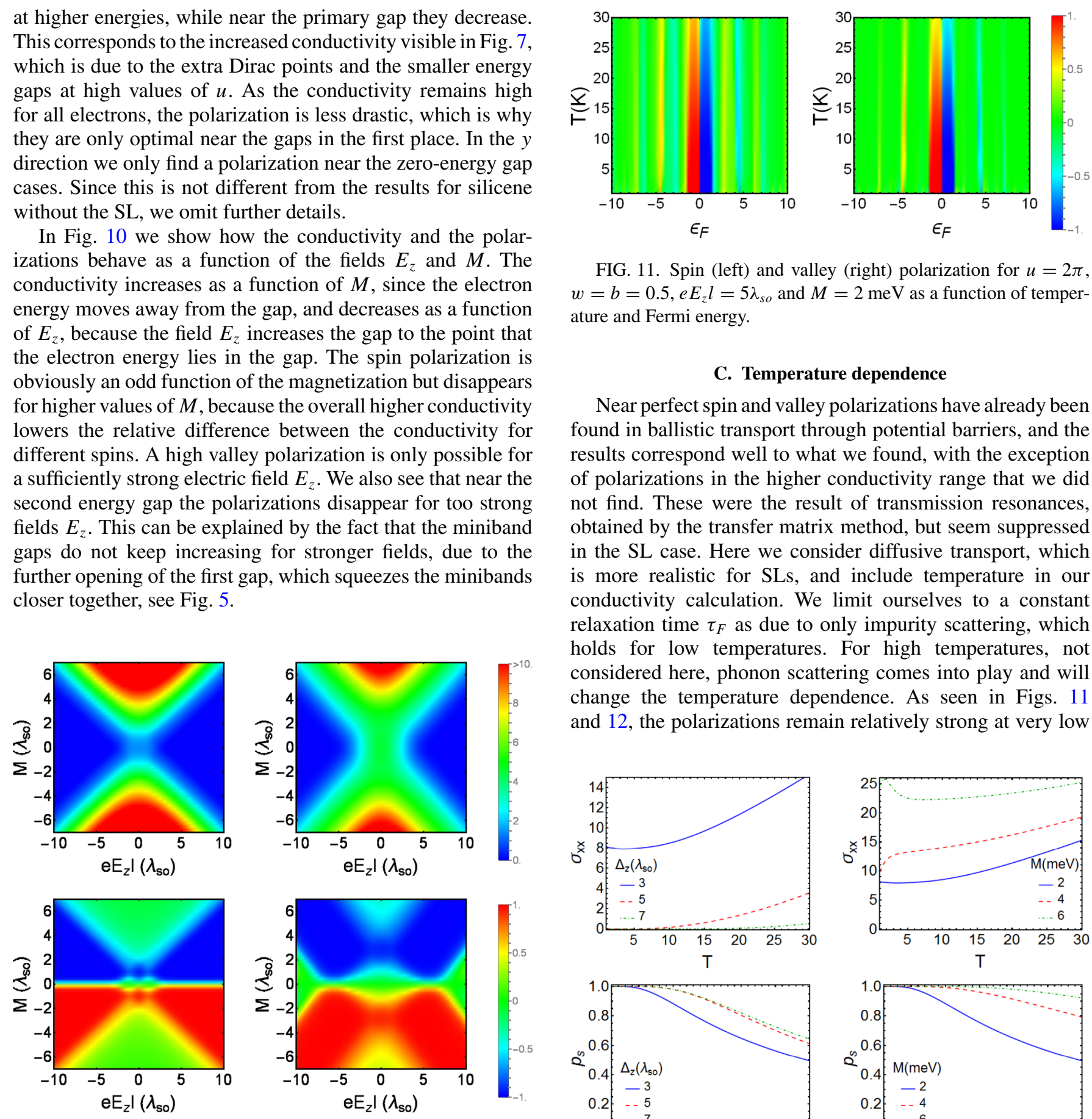

FIG. 11. Spin (left) and valley (right) polarization for $u=2 \pi$, $w=b=0.5, e E_{z} l=5 \lambda_{s o}$ and $M=2 \mathrm{meV}$ as a function of temperature and Fermi energy.

\section{Temperature dependence}

Near perfect spin and valley polarizations have already been found in ballistic transport through potential barriers, and the results correspond well to what we found, with the exception of polarizations in the higher conductivity range that we did not find. These were the result of transmission resonances, obtained by the transfer matrix method, but seem suppressed in the SL case. Here we consider diffusive transport, which is more realistic for SLs, and include temperature in our conductivity calculation. We limit ourselves to a constant relaxation time $\tau_{F}$ as due to only impurity scattering, which holds for low temperatures. For high temperatures, not considered here, phonon scattering comes into play and will change the temperature dependence. As seen in Figs. 11 and 12 , the polarizations remain relatively strong at very low
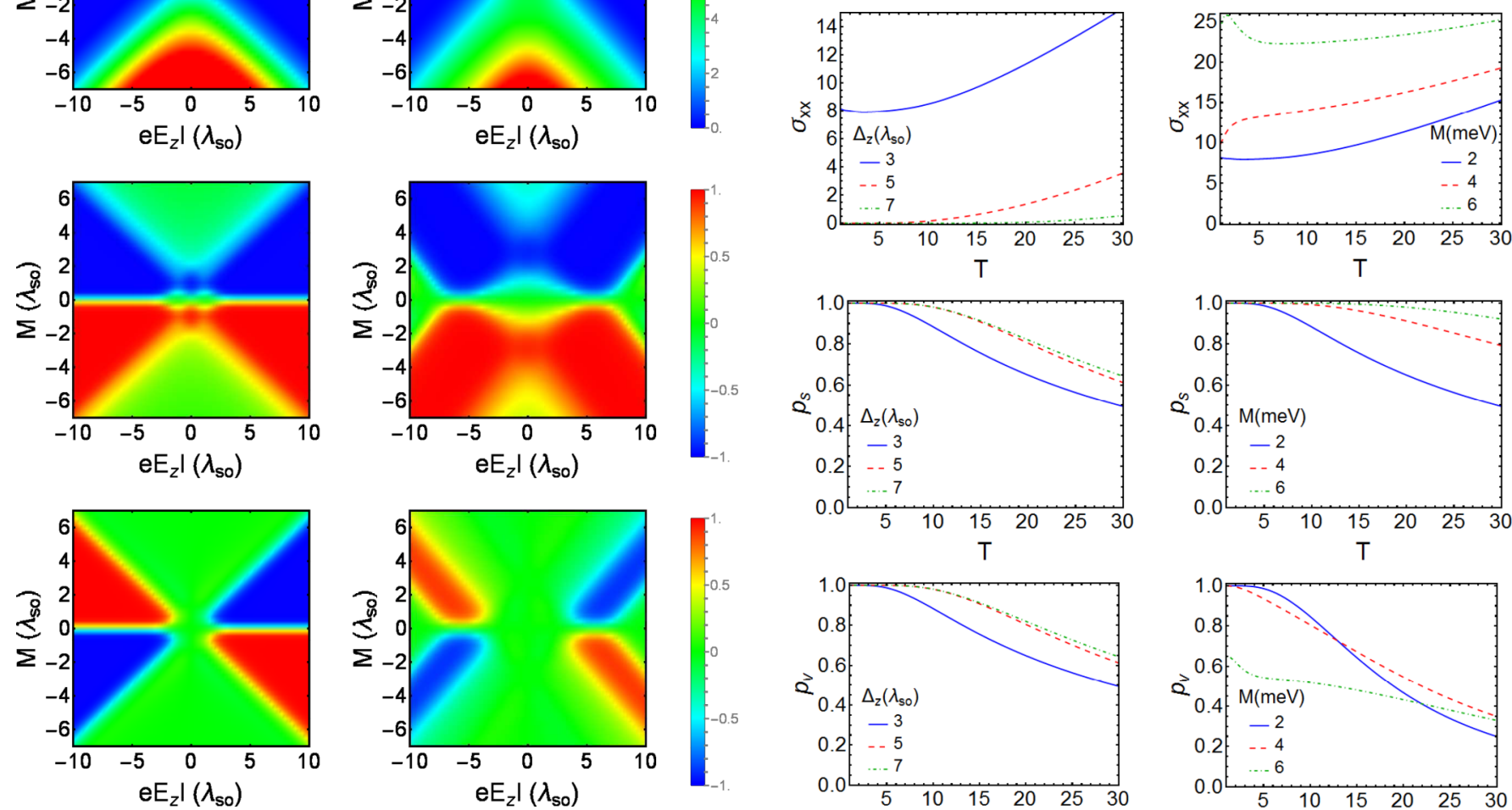

FIG. 10. $\left(M, E_{z}\right)$ contour plots of the conductivity in units of $4 \sigma_{0}$ (top), of the spin polarization (middle), and of the valley polarization (bottom) at $T=10 \mathrm{~K}$. The left panels are for $\epsilon_{F}=0.3$ and $u=2 \pi$ and the right ones for $\epsilon_{F}=\pi+0.3$ and $u=4 \pi$.
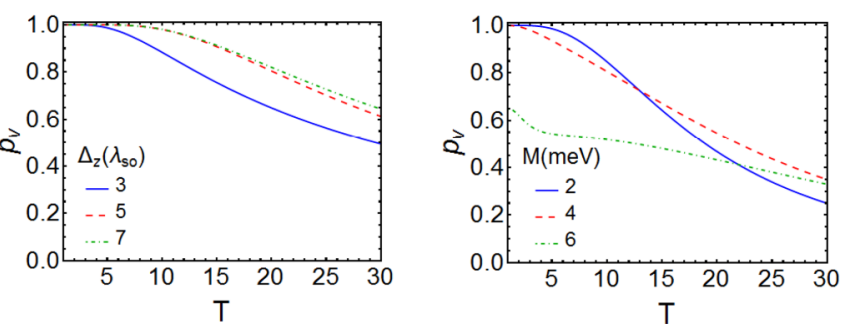

FIG. 12. Conductivity $\sigma_{x x}, \operatorname{spin}\left(p_{s}\right)$ and valley $\left(p_{v}\right)$ polarizations versus temperature for $u=2 \pi, w=b=0.5$. The left panels are for $M=2$ and the right ones for $e E_{z} l=3 \lambda_{s o}$. 
temperatures. Their decrease with temperature can be offset by using stronger fields. If the fields are too high, however, the total electron energy, in which the polarizations are optimal, will lie outside the narrow energy region, see Fig. 10. The conductivity increases with temperature because the smearing of the energy bands allows more energy states to contribute to the conduction.

\section{SUMMARY AND CONCLUSIONS}

We investigated the behavior of the energy gaps in silicene SLs as a function of the applied electric $E_{z}$ and exchange $M$ fields. Initially, the field $E_{z}$ creates energy band gaps, but due to the presence of extra Dirac points on both sides of the $K$ and $K^{\prime}$ valleys, the gaps close again when the Dirac points move towards the origin of the $K\left(K^{\prime}\right)$ valleys. Before all extra Dirac points annihilate in this way, there are zero-energy modes and the system remains metallic, contrary to the case of silicene without a SL. The gaps between the minibands behave in a similar fashion, although for large $E_{z}$, these gaps diminish in size. This is a consequence of the widening of the first band gap, which pushes the minibands closer together.

From the energy spectrum we obtained the DOS and the longitudinal conductivity in the $x$ and $y$ directions. Since both depend on the spin and valley degrees of freedom when the fields $E_{z}$ and $M$ are present, the different conductivities can be used to calculate the spin and valley polarizations. At very low temperatures we find nearly perfect polarizations close to the different gaps in the miniband structure. The polarizations above and below the gaps are opposite to each other. Near which gaps they are optimal depends on the potential strength $u$. For higher $u$ the polarizations near the gaps at higher energies are improved, while at lower energies the extra Dirac points keep the conductivity high for all electrons, thus decreasing the polarization.

The polarizations decrease with increasing temperature but this decrease can be partially offset by increasing the strength of the fields $E_{z}$ and $M$. Compared to the results of the transfermatrix method for a finite number of barriers, the general behavior of the polarizations versus the applied fields is similar, with the exception of polarizations in the higher conductivity range [24] which resulted from resonances in the transmission probability but which are suppressed in the SL case. However, due to the SL miniband structure highly efficient polarizations are found for a wider range of electron energies.

\section{ACKNOWLEDGMENTS}

This work was supported by the Canadian NSERC Grant No. OGP0121756 (P.V.), and by the Flemish Science Foundation FWO-Vl) with the "Odysseus" Program (N. M.) and with a $\mathrm{PhD}$ research grant (B.V.D.).
[1] Z. Ni, Q. Liu, K. Tang, J. Zheng, J. Zhou, R. Qin, Z. Gao, D. Yu, and J. Lu, Nano Lett. 12, 113 (2012); Y. Cai, C.-P. Chuu, C. M. Wei, and M. Y. Chou, Phys. Rev. B 88, 245408 (2013); M. Neek-Amal, A. Sadeghi, G. R. Berdiyorov, and F. M. Peeters, Appl. Phys. Lett. 103, 261904 (2013).

[2] G. G. Guzmán-Verri and L. C. Lew Yan Voon, Phys. Rev. B 76, 075131 (2007); S. Lebègue and O. Eriksson, ibid. 79, 115409 (2009).

[3] P. Vogt, P. De Padova, C. Quaresima, J. Avila, E. Frantzeskakis, M. C. Asensio, A. Resta, B. Ealet, and G. Le Lay, Phys. Rev. Lett. 108, 155501 (2012); A. Fleurence, R. Friedlein, T. Ozaki, H. Kawai, Y. Wang, and Y. Yamada-Takamura, ibid. 108, 245501 (2012).

[4] D. Chiappe, E. Scalise, E. Cinquanta, C. Grazianetti, B. v. Broek, M. Fanciulli, M. Houssa, and A. Molle, Adv. Mater. 26, 2096 (2014).

[5] L. Chen, C.-C. Liu, B. Feng, X. He, P. Cheng, Z. Ding, S. Meng, Y. Yao, and K. Wu, Phys. Rev. Lett. 109, 056804 (2012).

[6] L. Meng, Y. Wang, L. Zhang, S. Du, R. Wu, L. Li, Y. Zhang, G. Li, H. Zhou, W. A. Hofer, and H. J. Gao, Nano. Lett. 13, 685 (2013).

[7] C.-C. Liu, H. Jiang, and Y. Yao, Phys. Rev. B 84, 195430 (2011).

[8] N. D. Drummond, V. Zólyomi, and V. I. F'alko, Phys. Rev. B 85, 075423 (2012).

[9] M. Ezawa, New. J. Phys. 14, 033003 (2012).

[10] L. Tao, E. Cinquanta, D. Chiappe, C. Grazianetti, M. Fanciulli, M. Dubey, A. Molle, and D. Akinwande, Nat. Nanotechnology 10, 227 (2015)

[11] B. Huang, D. J. Monsma, and I. Appelbaum, Phys. Rev. Lett. 99, 177209 (2007).

[12] Y. Wang, J. Zheng, Z. Ni, R. Fei, Q. Liu, R. Quhe, C. Xu, J. Zhou, Z. Gao, and J. Lu, Nano 07, 1250037 (2012).
[13] S. Sanvito, Chem. Soc. Rev. 40, 3336 (2011).

[14] N. Tombros, C. Jozsa, M. Popinciuc, H. T. Jonkman, and B. J. van Wees, Nature (London) 448, 571 (2007).

[15] C.-C. Liu, W. Feng, and Y. Yao, Phys. Rev. Lett. 107, 076802 (2011).

[16] A. Dyrdał and J. Barnas, Phys. Stat. Sol. RRL 6, 340 (2012).

[17] M. Tahir, A. Manchon, K. Sabeeh, and U. Schwingenschlögl, Appl. Phys. Lett. 102, 162412 (2013).

[18] C. J. Tabert and E. J. Nicol, Phys. Rev. B 87, 235426 (2013).

[19] M. Ezawa, Phys. Rev. Lett. 109, 055502 (2012).

[20] L. Stille, C. J. Tabert, and E. J. Nicol, Phys. Rev. B 86, 195405 (2012).

[21] A. Kara, H. Enriquez, A. P. Seitsonen, L. C. Lew Yan Voon, S. Vizzini, B. Aufray, and H. Oughaddoub, Surf. Sci. 67, 1 (2012).

[22] T. Yokoyama, Phys. Rev. B 87, 241409(R) (2013).

[23] V. Vargiamidis and P. Vasilopoulos, Appl. Phys. Lett. 105, 223105 (2014); J. Appl. Phys. 117, 094305 (2015).

[24] N. Missault, P. Vasilopoulos, V. Vargiamidis, F. M. Peeters, and B. Van Duppen, Phys. Rev. B 92, 195423 (2015).

[25] C.-H. Park, Y.-W. Son, L. Yang, M. L. Cohen, and S. G. Louie, Phys. Rev. Lett. 103, 046808 (2009); L. Brey and H. A. Fertig, ibid. 103, 046809 (2009).

[26] M. Barbier, P. Vasilopoulos, and F. M. Peeters, Phys. Rev. B 81, 075438 (2010); Phil. Trans. R. Soc. A 368, 5499 (2010).

[27] B. Van Duppen, P. Vasilopoulos, and F. M. Peeters, Phys. Rev. B 90, 035142 (2014).

[28] M. Charbonneau, K. M. van Vliet, and P. Vasilopoulos, J. Math. Phys. 23, 318 (1982).

[29] V. Vargiamidis, P. Vasilopoulos, and G. Q. Hai, J. Phys. Condens. Matter 26, 345303 (2014) 